\title{
Percepção auditiva de professores disfônicos
}

\section{Auditory perception of teachers with voice disorders}

\author{
Mara Mércia Belúcio Buosi', Léslie Piccolotto Ferreira², Teresa Maria Momensohn-Santos³
}

\section{RESUMO}

Objetivo: Descrever os achados da avaliação de habilidades auditivas - traços de frequência, intensidade e duração - em um grupo de professores disfônicos (GD), comparando-os aos encontrados em professores não-disfônicos (GND). Métodos: Foram avaliados 44 sujeitos do gênero feminino, na faixa etária de 18 a 40 anos, sendo 23 disfônicos (GD) e 21 não-disfônico (GND). Para definir esses grupos, as professoras foram submetidas à avaliação laringológica e perceptivo-auditiva da voz, bem como, à avaliação audiológica completa, a fim de que fossem excluídas aquelas que apresentassem perda auditiva. Após a seleção, procedeu-se a investigação do Limiar Diferencial de Intensidade e da aplicação dos Testes de Detecção de Padrão de Frequência e Duração. Resultados: O GND apresentou melhor desempenho que o GD na avaliação do parâmetro de frequência. Conclusão: A análise relacionada à percepção auditiva mostra diferença apenas para o parâmetro da frequência entre professores disfônicos e não disfônicos. Estudos com maior número de sujeitos e com outros testes que avaliem o limiar diferencial de intensidade podem esclarecer melhor a relação entre problemas de voz e dificuldades na percepção de pequenas diferenças de intensidade.

Descritores: Disfonia; Docentes; Percepção auditiva; Voz; Audição

\begin{abstract}
Purpose: To describe the findings of the assessment of auditory skills frequency, intensity and duration patterns - in a group of teachers with voice disorders (DG), and to compare the findings in a group of teachers without vocal disorders (NDG). Methods: There were 44 females, between 18 and 40 years of age, 23 were placed in the voice disorder group (DG) and 21 in the group without voice disorders (NDG). In order to define these groups, the teachers were submitted to both vocal behavior and laryngeal assessments, as well as to a complete audiological evaluation in order to assure that those subjects with hearing loss were excluded from the study. Following subject selection, an investigation of the Differential Intensity Threshold was carried out, along with the administration of the tests for Frequency and Duration Pattern Detection. Results: The NDG had better performance than the DG in all administered tests, with a significant difference in the evaluation of the frequency parameter. Conclusion: Analysis of the performance of the two groups of teachers showed statistically significant only for the frequency parameter. Studies with larger numbers of subjects and with other tests that evaluate the differential threshold of intensity may perhaps clarify better the relationship between voice problems and difficulties in the realization of small differences in intensity.
\end{abstract}

Keywords: Dysphonia; Faculty; Auditory perception; Voice; Hearing

Trabalho realizado na Clínica-escola de Fonoaudiologia das Faculdades Integradas de Fernandópolis - FIFE - Fernandópolis (SP), Brasil.

(1) Curso de Fonoaudiologia, Faculdades Integradas de Fernandópolis - FIFE - Fernandópolis (SP), Brasil.

(2) Departamento de Fundamentos da Fonoaudiologia, Pontifícia Universidade Católica de São Paulo - PUC - São Paulo (SP), Brasil; Programa de Estudos Pós-Graduados em Fonoaudiologia, Pontifícia Universidade Católica de São Paulo - PUC - São Paulo (SP), Brasil.

(3) Departamento de Clínica Fonoaudiológica, Pontifícia Universidade Católica de São Paulo - PUC - São Paulo (SP), Brasil; Instituto de Estudos da Audição

- IEAA - São Paulo (SP), Brasil.

Conflito de interesses: Não

Contribuição dos autores: $M M B B$ realizou busca à literatura, coleta de dados, análise e interpretação dos dados, redação do manuscrito; $L P F$ e $T M M S$ orientaram a pesquisa.

Endereço para correspondência: Mara Mércia Belúcio Buosi. Av. Teotônio Vilela s/nº Campus Universitário, Fernandópolis (SP), Brasil, CEP: 15600-000.

E-mail: marafono@fef.br

Recebido em: 13/6/2011; Aceito em: 26/2/2013 


\section{INTRODUÇÃO}

Dois estudos realizados com professores certificam a alta ocorrência de distúrbio vocal entre esses profissionais. Uma pesquisa realizada nos Estados Unidos com docentes e não docentes evidenciou maior ocorrência de distúrbios vocais em professores (57,7\% dos entrevistados afirmaram ter algum tipo de distúrbio vocal ao longo de sua carreira profissional) em comparação aos profissionais não docentes $(28,8 \%)^{(1)}$. Em um estudo, de origem brasileira, baseado na mesma metodologia do anterior, o distúrbio da voz foi referido por $66,7 \%$ dos professores, enquanto apenas $14,8 \%$ dos não professores indicaram a presença de sintomas no mesmo momento ${ }^{(2)}$. Estudos evidenciam que a falta de conhecimento acerca da própria voz e a dificuldade de autopercepção, por serem medidas subjetivas, podem explicar a alta incidência desse distúrbio entre os professores $^{(3,4)}$.

A voz e a audição são aspectos que se relacionam. Ouvir é uma atividade complexa e elaborada, que envolve desde a detecção de estímulos sonoros pelos órgãos periféricos, até sua interpretação pelo sistema nervoso auditivo central. Dessa forma, a audição se constitui em sentido importante para o monitoramento de um padrão vocal adequado. Para que se tenha uma boa voz, no mínimo, é preciso saber ouví-la ${ }^{(4)}$, principalmente para o profissional da voz, como é o caso do professor.

As queixas vocais dos professores parecem estar relacionadas a um conjunto de fatores. São comumente relatados ardor e dor ao falar, tosses, infecções de laringe, rouquidão e perda total da voz ${ }^{(5-9)}$. Esses fatores geralmente estão relacionados ao ambiente ruidoso ${ }^{(10)}$, que conduz o professor a adotar, mesmo sem perceber, uma voz em intensidade aumentada e frequência agudizada ${ }^{(11-13)}$. A exposição ao ruído exige do professor uma demanda vocal abusiva ${ }^{(14)}$ e essa é uma das queixas mais frequentes entre eles ${ }^{(15,16)}$.

Dessa forma, quando o indivíduo usa a voz profissionalmente, é importante que passe por uma avaliação dos limiares audiométricos e reconhecimento das palavras, pois seu monitoramento vocal será realizado basicamente pela via auditiva, que precisa estar preservada. Fica evidente que o ajuste motor empregado para que determinada qualidade vocal possa ser produzida depende do monitoramento feito pela pista da audição ${ }^{(17)}$, ou seja, é influenciado pelas habilidades auditivas.

A literatura propõe que se faça avaliação das habilidades auditivas dos disfônicos utilizando-se "sons grosseiros"(18), ou seja, sons que não apresentam características acústicas bem definidas. Diante de tal questão, decidiu-se buscar junto à Audiologia procedimentos formais que viabilizassem analisar as habilidades auditivas para discriminar traços de frequência, intensidade e duração. Desse modo, seria possível verificar o quanto esses indivíduos conseguiriam discriminar os três parâmetros acústicos da voz por meio de tons puros.

$\mathrm{Na}$ bateria de avaliação do processamento auditivo existem dois testes que avaliam a habilidade de um indivíduo em identificar amostras de tons puros em duas diferentes condições. Um dos testes avalia a habilidade de detectar sequências de um som, de uma mesma frequência, em duas diferentes durações. Este teste foi denominado Teste de Detecção de Padrão de Duração ${ }^{(19)}$.

O outro teste, denominado Teste de Detecção de Padrão de Frequência ${ }^{(19)}$, obedece ao mesmo modelo de construção, porém nessa prova os estímulos se diferenciam pela frequência. Assim, as amostras de tom apresentam as características acústicas de tons de $1430 \mathrm{~Hz}$ (Agudo - A) e $880 \mathrm{~Hz}$ (Grave - G), com duração de $200 \mathrm{~ms}$.

O teste de Índice de Sensibilidade a Pequenos Incrementos $(\mathrm{SISI})^{(20)}$ tem como objetivo medir a capacidade da orelha em detectar pequenas alterações de intensidade. Em pesquisa realizada com um grupo controle (GC) e outro de disfônicos (GD) com alterações vocais funcionais ${ }^{(21)}$, o autor aplicou uma avaliação dos processos temporais compostos por três subtestes: Discriminação Auditiva (DA), Reconhecimento da Mudança de Tom (RMT) e Reconhecimento do Padrão de Frequência (RPF). Os resultados mostraram que os indivíduos com disfonia funcional tinham maior dificuldade em responder a esses testes de processos temporais auditivos. Diante de tais achados, o autor recomendou que se realizasse a avaliação dos processos temporais da audição em indivíduos que apresentassem ou não disfonias funcionais, com ou sem prejuízo da capacidade de detecção de sons, com a finalidade de investigar o processamento de sons não verbais para a automonitorização vocal.

Dessa forma, as habilidades auditivas e a produção da voz não podem ser vistas de maneira dicotomizada, pois ambas interagem e pertencem a um mesmo sujeito. A audição analisa os parâmetros acústicos que a voz produz, fato esse que evidencia a importância de se analisar a audição do ponto de vista perceptual. Para entender melhor e dessa forma poder planejar o modelo de intervenção mais adequado a cada caso, torna-se necessário investigar como o paciente "se ouve" e levantar questões acerca de quais as consequências do "não se ouvir" para a monitorização vocal. Em outras palavras, se o paciente apresenta dificuldades para identificar e discriminar um dos parâmetros acústicos da voz (frequência, intensidade ou duração), isto poderia se configurar como uma justificativa para o processo terapêutico não evoluir. A integridade da percepção auditiva é primordial para a fala, para a compreensão da linguagem e para o aprendizado. A identificação da natureza do prejuízo auditivo contribui para direcionamento da intervenção. A voz, quando produzida em intensidade e projeção adequadas, proporciona um bom desempenho vocal para o professor $^{(3)}$.

Para responder à questão da interação entre habilidades perceptuais auditivas e produção de voz, o objetivo deste trabalho foi descrever os achados de avaliação de habilidades auditivas - traços de frequência, intensidade e duração - em grupo de professores disfônicos (GD), comparando-os aos encontrados em professores não disfônicos (GND). 


\section{MÉTODOS}

Os sujeitos desta pesquisa foram professores, de ensino fundamental a superior, das redes pública e particular do município de Fernandópolis, São Paulo, Brasil. A pesquisa foi aprovada pelo Comitê de Ética da Pontifícia Universidade Católica de São Paulo (processo n ${ }^{\circ} 0047$ ).

Inicialmente foram realizadas palestras com os professores, com o objetivo de selecionar aqueles que tivessem interesse em participar deste estudo. Compareceram às palestras 82 professores. Os interessados assinaram Termo de Consentimento Livre e Esclarecido e foram informados a respeito da justificativa e objetivo da pesquisa, tomando ciência dos critérios pré-estabelecidos para sua seleção, bem como dos procedimentos a que seriam submetidos.

Os critérios de inclusão foram: ter entre 18 a 40 anos (período de eficiência vocal); ser brasileiro, tendo o português como língua materna; não ter realizado terapia fonoaudiológica; não relatar distúrbios de natureza endocrinológica, neurológica ou auditiva. Essas condições foram garantidas por meio de autorreferência dos sujeitos, a partir de aplicação de questionário. Dessa forma, foram selecionados 50 interessados.

Posteriormente, os sujeitos submeteram-se a:

avaliação de laringe: realizada por meio de nasoendoscópio e tele laringoscópio (nasoendoscópio Machida® 3,2 mm, telelaringoscópio ASAP $70^{\circ}$, micro câmera e videocassete). O exame foi realizado em consultório otorrinolaringológico, por dois otorrinolaringologistas, acompanhados por uma fonoaudióloga. Ao final, com base nos achados, determinava-se a presença ou ausência de alterações na laringe; avaliação perceptivo-auditiva da voz: foi solicitado aos professores que emitissem as vogais sustentadas /a, /i/, e /u/ e uma sequência de contagem de números. As amostras foram gravadas (gravador Aiwa ${ }^{\circledR}$ TP-VS 535) para posterior análise por meio da Escala GRBASI ${ }^{(22)}$. As vozes foram avaliadas inicialmente de acordo com o grau de alteração geral ( $\mathrm{G}$ - grade), de 0 a 3 (ausência de alteração=0, alteração discreta $=1$, alteração moderada $=2$, alteração extrema=3), e na sequência, quanto à presença de rugosidade (roughness), soprosidade (breathiness), astenia (asthenicity), tensão (S strain) e instabilidade (I - instability), parâmetros também avaliados nos mesmos graus anteriormente descritos. Ao final, os sujeitos que apresentaram desvio vocal em grau geral maior que um foram considerados como apresentando alteração perceptivo-auditiva de voz.

audiometria tonal: os indivíduos foram submetidos à inspeção da orelha externa, sendo que os sujeitos que apresentaram obstrução do meato acústico externo foram orientados a procurar por um serviço médico para limpeza e, posteriormente, marcar novo dia para avaliação. A audiometria tonal foi realizada em cabina acústica, utilizando-se audiômetro calibrado segundo o padrão ANSI-69. Foram avaliadas as frequências de $250 \mathrm{~Hz}$ a $8000 \mathrm{~Hz}$ com os sujeitos posicionados de costas para o audiômetro;

- pesquisa do limiar de reconhecimento da fala, ou Speech Recognition Threshold (SRT) e pesquisa do índice de reconhecimento de fala ${ }^{(20)}$;

- medidas da imitância acústica: realizadas por meio do analisador de orelha média Interacoustics $® A Z 7^{(20)}$.

Embora a situação ideal fosse conciliar, em um único momento, a realização dos procedimentos audiológicos, exame laringológico e de voz, isto não foi possível em função da dificuldade de disponibilidade de horário das professoras. Após a realização dos procedimentos audiológicos, seis professoras foram excluídas, e as outras 44 foram distribuídas, a partir da avaliação de laringe e de voz, em dois grupos:

- Grupo não-disfônico (GND): composto por 21 professoras que não apresentaram alterações na avaliação de laringe e/ou de voz. A média de idade desse grupo foi de 28,23 (mínimo de 20 e máximo de 36 anos). Desse grupo, seis professoras ministravam aulas no Ensino Superior, quatro no Ensino Médio,cinco no Ensino Fundamental,e seis na Educação Infantil.

- Grupo disfônico (GD): composto por 23 professoras que apresentaram alteração nas avaliações de laringe (15 casos de fenda triangular médio-posterior, sete casos de nódulos vocais e um caso de fonação vestibular) e de voz (12 apresentaram grau global de disfonia de nível moderado e 11 de nível intenso). A média de idade desse grupo foi de 30,52 (mínimo de 21 e máximo de 37 anos), sendo que cinco ministravam aulas no Ensino Superior, sete no Ensino Médio, seis no Ensino Fundamental e cinco na Educação Infantil.

Depois de definidos os dois grupos, todos participantes foram avaliados quanto às habilidades auditivas. Para a referida avaliação, foram utilizados os Testes de Detecção de Padrão de Frequência e de Duração e a Pesquisa do Limiar Diferencial de Intensidade.

Os Testes de Detecção de Padrão de Duração e de Frequência foram executados em meio ambiente silencioso, na condição binaural, utilizando-se CD player marca Sony® e CD da Auditec de St. Louis EUA (1997). A Pesquisa do Limiar Diferencial de Intensidade foi realizada utilizando-se audiômetro GSI-61, com fone TDH-50P e coxim B-17 calibrado segundo o padrão ANSI-69.

O Teste de Detecção de Padrão de Duração contem 60 amostras (seis padrões apresentados aleatoriamente 10 vezes). Os tons são de $1000 \mathrm{~Hz}$, com tempo de ataque e recuperação de $10 \mathrm{~ms}$. O tom longo (L) tem duração de $500 \mathrm{~ms}$ e o curto (C) de $250 \mathrm{~ms}^{(19)}$. A participante foi instruída a imitar ou nomear a sequência de três tons, obedecendo a ordem de apresentação: LLC; CCL; LCL e assim por diante. Conforme consta do manual de aplicação do teste ${ }^{(19)}$ são esperados resultados acima de $87 \%$ de acertos.

O teste de Padrão de Frequência é composto por 60 sequências de amostras, cada uma delas composta por três tons. 
Tais sequências são organizadas da seguinte forma: GGA; AGG; AGA; GAG; AAG; GAA. Nesta prova, a participante foi orientada a imitar ou nomear as sequências que ouvia na ordem em que elas foram apresentadas. Para este teste o manual estabelece que o sujeito deva acertar acima de $90 \%$ dos itens apresentados.

$\mathrm{Na}$ pesquisa do Limiar Diferencial de Intensidade, por meio do teste SISI, o tom de base foi apresentado para ao indivíduo em um nível de 20 dBNS. A cada 5 segundos, foi sobreposto um pequeno incremento, iniciando-se com incremento de $5 \mathrm{~dB}$. O sinal tem um tempo de duração de $50 \mathrm{~ms}$ e um intervalo de 5 segundos entre cada incremento. A participante foi instruída a indicar se ouvia um breve "aumento" na intensidade do tom. Depois de aproximadamente cinco incrementos, a magnitude do incremento foi reduzida a $1 \mathrm{~dB}$, marcando o início da contagem do teste SISI. Foram apresentados 20 incrementos de $1 \mathrm{~dB}$, e solicitado à professora que indicasse quando ouvia cada incremento. Se forem ouvidos vários incrementos consecutivos (aproximadamente cinco), o examinador retardava alguns incrementos de modo que ele pudesse determinar se o indivíduo estava respondendo à alteração de intensidade ou a um intervalo de tempo aprendido. Se o indivíduo não respondesse a vários incrementos sucessivos, a magnitude do incremento era aumentada para novo treinamento, para dar a oportunidade de centrar a atenção novamente na tarefa antes do teste com incrementos de $1 \mathrm{~dB}$. Assim, as respostas do tipo falso-negativo e falso-positivo puderam ser minimizadas. O procedimento recomendado, comum na prática clínica, inicia-se com incrementos de $5 \mathrm{~dB}$ e, tendo obtido respostas, reduz-se a magnitude do incremento para $4,3,2$, e, finalmente, $1 \mathrm{~dB}$, antes de iniciar a contagem para o teste ${ }^{(20)}$.

Para análise, as respostas dos três testes aplicados foram digitadas em planilha especifica e utilizado o programa estatístico SPSS versão 10.0. A parte destinada à descrição da amostra constou dos cálculos de medidas-resumo, a saber: média aritmética simples, desvio-padrão e mediana de cada uma das variáveis do estudo.

A análise estatística constou das seguintes etapas: para a comparação entre as orelhas direita e esquerda, para os resultados do SISI, foi usado o teste dos Postos Sinalizados de Wilcoxon; esse teste foi aplicado para os dois grupos, em separado; para a comparação entre os dois grupos, para os resultados do SISI, foi usado o teste de Mann-Whitney; e para a comparação entre os grupos, para as variáveis dos blocos TDPF e TDPD, foi usado o teste $t$ de Student. Para todas as análises foi adotado nível de significância de 5\% ( $\leq \leq 0,050)$.

\section{RESULTADOS}

Os grupos GND e GD mostrara-se semelhantes em relação à média de idade, distribuição por nível de ensino e disciplinas ministradas. Houve, assim, homogeneidade entre eles.

\section{Análise do desempenho nos testes de detecção de padrão de frequência e duração}

Foi observado desempenho superior do GND em relação ao GD no Teste de Detecção de Padrão de Frequência (TDPF) (Tabela 1). No que se refere ao Teste de Detecção de Padrão de Duração (TDPD), em termos percentuais, pode-se dizer que não houve diferenças (Tabela 2).

Foi realizada ainda a comparação entre GND e GD quanto aos índices de acertos, tanto para o Teste de Detecção de Padrão de Frequência (TDPF) quanto para o Teste de Detecção de Padrão de Duração (TDPD) (Tabela 3).

\section{Análise do desempenho na pesquisa do limiar diferencial de intensidade}

Na comparação entre os grupos, por orelha, diferenças foram notadas (Tabela 4), a saber: na OD, nas frequências de 500 $\mathrm{Hz}(20$ incrementos de $1 \mathrm{~dB}), 1000 \mathrm{~Hz}$ ( 5 incrementos de $5 \mathrm{~dB}$ e 20 incrementos de $1 \mathrm{~dB}), 2000 \mathrm{~Hz}$ ( 20 incrementos de $1 \mathrm{~dB}$ ) e $4000 \mathrm{~Hz}$ (20 incrementos de $1 \mathrm{~dB}$ ); e, na OE, nas frequências de $500 \mathrm{~Hz}$ ( 5 incrementos de $2 \mathrm{~dB}$ e 20 incrementos de $1 \mathrm{~dB}$ ), $1000 \mathrm{~Hz}$ ( 5 incrementos de $5 \mathrm{~dB}$ e 20 incrementos de $1 \mathrm{~dB}$ ), $2000 \mathrm{~Hz}$ ( 5 incrementos de $5 \mathrm{~dB}$ e 20 incrementos de $1 \mathrm{~dB}$ ) e $4000 \mathrm{~Hz}$ (20 incrementos de $1 \mathrm{~dB}$ ).

$\mathrm{Na}$ análise estatística, ao ser estabelecida a comparação entre OD e OE por grupo de estudo, observa-se que no GD houve diferença na frequência de $2000 \mathrm{~Hz}$ (apresentação de 5 incrementos de $2 \mathrm{~dB}$ ), e na frequência de $4000 \mathrm{~Hz}$ (apresentação de 20 incrementos de $2 \mathrm{~dB}$ ) (Tabela 5).

\section{DISCUSSÃO}

Considerando-se a dificuldade de encontrar pesquisas semelhantes a esta, para a discussão dos achados foram utilizados autores que mostrassem alguma afinidade com nossa proposta, ou que utilizaram os mesmos procedimentos.

$\mathrm{Na}$ avaliação do parâmetro de frequência, na qual foi empregado o Teste de Detecção de Padrão de Frequência (TDPF), os valores obtidos pelos dois grupos estão de acordo com o proposto pela literatura ${ }^{(19)}$. Estudos propõem como esperados valores acima de $76 \%{ }^{(23)}$. Portanto, ao se comparar o desempenho dos grupos, tendo como referência esses valores, verifica-se que o GND apresentou melhor desempenho do que o GD. Desta forma, apesar de a maioria dos indivíduos do GD apresentar valores dentro do esperado, nota-se que o desempenho deles foi inferior ao dos participantes do GND.

Em relação ao Teste de Deteç̧ão de Padrão de Duração (TDPD), estudos propuseram como resultados esperados valores acima de $87 \%^{(19)}$ e $83 \%{ }^{(23)}$. Portanto, de acordo com os valores propostos na literatura há evidências de que o desempenho do GND foi superior ao do GD.

Demanda vocal, fatores sócio demográficos e riscos 
Tabela 1. Distribuição numérica e percentual de acertos e erros obtidos na avaliação do parâmetro de frequência nos grupos não-disfônico e disfônico

\begin{tabular}{|c|c|c|c|c|c|c|c|c|c|}
\hline \multirow{3}{*}{ Sujeito } & \multicolumn{4}{|c|}{ GND } & \multirow{3}{*}{ Sujeito } & \multicolumn{4}{|c|}{ GD } \\
\hline & \multicolumn{2}{|c|}{ Acertos } & \multicolumn{2}{|c|}{ Erros } & & \multicolumn{2}{|c|}{ Acertos } & \multicolumn{2}{|c|}{ Erros } \\
\hline & $\mathrm{N}$ & $\%$ & $\mathrm{~N}$ & $\%$ & & $\mathrm{~N}$ & $\%$ & $\mathrm{~N}$ & $\%$ \\
\hline 01 & 54 & 90,00 & 6 & 10,00 & 01 & 58 & 96,67 & 2 & 3,33 \\
\hline 02 & 59 & 98,34 & 1 & 1,66 & 02 & 53 & 88,33 & 7 & 11,67 \\
\hline 03 & 58 & 96,67 & 2 & 3,33 & 03 & 45 & 75,00 & 15 & 25,00 \\
\hline 04 & 58 & 96,67 & 2 & 3,33 & 04 & 57 & 95,00 & 3 & 5,00 \\
\hline 05 & 60 & 100,00 & 0 & 0,00 & 05 & 58 & 96,67 & 2 & 3,33 \\
\hline 06 & 60 & 100,00 & 0 & 0,00 & 06 & 58 & 96,67 & 2 & 3,33 \\
\hline 07 & 60 & 100,00 & 0 & 0,00 & 07 & 55 & 91,67 & 5 & 8,33 \\
\hline 08 & 60 & 100,00 & 0 & 0,00 & 08 & 53 & 88,33 & 7 & 11,67 \\
\hline 09 & 54 & 90,00 & 6 & 10,00 & 09 & 57 & 95,00 & 3 & 5,00 \\
\hline 10 & 55 & 91,67 & 5 & 8,33 & 10 & 57 & 95,00 & 3 & 5,00 \\
\hline 11 & 60 & 100,00 & 0 & 0,00 & 11 & 58 & 96,67 & 2 & 3,33 \\
\hline 12 & 58 & 96,67 & 2 & 3,33 & 12 & 58 & 96,67 & 2 & 3,33 \\
\hline 13 & 60 & 100,00 & 0 & 0,00 & 13 & 58 & 96,67 & 2 & 3,33 \\
\hline 14 & 58 & 96,67 & 2 & 3,33 & 14 & 59 & 98,33 & 1 & 1,67 \\
\hline 15 & 58 & 96,67 & 2 & 3,33 & 15 & 56 & 93,33 & 4 & 6,67 \\
\hline 16 & 60 & 100,00 & 0 & 0,00 & 16 & 57 & 95,00 & 3 & 5,00 \\
\hline 17 & 59 & 98,34 & 1 & 1,66 & 17 & 60 & 100,00 & 0 & 0,00 \\
\hline 18 & 59 & 98,34 & 1 & 1,66 & 18 & 59 & 98,33 & 1 & 1,67 \\
\hline 19 & 60 & 100,00 & 0 & 0,00 & 19 & 47 & 78,33 & 13 & 21,67 \\
\hline 20 & 60 & 100,00 & 0 & 0,00 & 20 & 53 & 88,33 & 7 & 11,67 \\
\hline \multirow[t]{3}{*}{21} & 60 & 100,00 & 0 & 0,00 & 21 & 60 & 100,00 & 0 & 0,00 \\
\hline & & & & & 22 & 56 & 93,33 & 4 & 6,67 \\
\hline & & & & & 23 & 60 & 100,00 & 0 & 0,00 \\
\hline
\end{tabular}

Legenda: GND = grupo não-disfônico; GD = grupo disfônico

diretamente relacionados à organização e ao ambiente de trabalho têm impacto na ocorrência de disfonia ${ }^{(9)}$. Nesse contexto, a percepção auditiva desempenha papel fundamental na busca do feedback vocal ${ }^{(24)}$, e a percepção auditiva dos parâmetros relacionados à voz tem influência direta sobre o ajuste motor a ser adotado. É sabido que a voz depende principalmente de uma complexa e interdependente atividade de todos os músculos que servem à sua produção, requerendo, portanto, um sofisticado processamento muscular. Dentro dessa perspectiva, acredita-se que o "não se ouvir" pode trazer consequências para a produção vocal, uma vez que o ajuste motor adotado poderá ser inadequado, fato que poderá interferir diretamente na evolução da reabilitação vocal.

O desempenho do GND e GD na avaliação do parâmetro de intensidade foi obtido mediante a determinação do Limiar Diferencial de Intensidade (SISI). Na Pesquisa do Limiar Diferencial de Intensidade, o GND apresentou, em algumas frequências, melhores resultados do que o GD. Com esse achado é possível hipotetizar que se o indivíduo não percebe as pequenas mudanças de intensidade que produz ao falar, ele poderá ter dificuldades para se monitorizar e não realizar as mudanças necessárias para não fazer uso de intensidade inadequada na voz, o que poderia gerará sobrecarga ao aparelho fonador, tornando-o mais suscetível a distúrbios vocais. Esta situação não tem a ver com o efeito, que ocorre quando o falante eleva a intensidade de sua voz, pois tem que competir com o nível sonoro do ruído presente no ambiente em que realiza seu discurso ${ }^{(25)}$.

O feedback acústico-articulatório ocorre a partir dos três meses de idade da criança, e é resultado da maturação do sistema auditivo central e de sua integração com outras áreas do cérebro. Um modelo interno que relaciona a configuração do trato vocal e o som a ser produzido é auxiliado a partir do feedback auditivo, que é utilizado para monitoramento das condições para que, também, haja possibilidade de reajustes nos parâmetros posturais pré-definidos ${ }^{(26)}$. O processamento sensorial auditivo é um importante elemento dos mecanismos neurais que controlam a vocalização humana.

Perturbações no padrão do feedback sensorial por si só, podem gerar expectativas imprecisas no sistema de controle 
Tabela 2. Distribuição numérica e percentual de acertos e erros obtidos na avaliação do parâmetro de duração nos grupos não-disfônico e disfônico

\begin{tabular}{|c|c|c|c|c|c|c|c|c|c|}
\hline \multirow{3}{*}{ Sujeito } & \multicolumn{4}{|c|}{ GND } & \multirow{3}{*}{ Sujeito } & \multicolumn{4}{|c|}{ GD } \\
\hline & \multicolumn{2}{|c|}{ Acertos } & \multicolumn{2}{|c|}{ Erros } & & \multicolumn{2}{|c|}{ Acertos } & \multicolumn{2}{|c|}{ Erros } \\
\hline & $\mathrm{N}$ & $\%$ & $\mathrm{~N}$ & $\%$ & & $\mathrm{~N}$ & $\%$ & $\mathrm{~N}$ & $\%$ \\
\hline 01 & 54 & 90,00 & 6 & 10,00 & 01 & 29 & 48,34 & 31 & 51,66 \\
\hline 02 & 56 & 93,34 & 4 & 6,66 & 02 & 27 & 45,00 & 33 & 55,00 \\
\hline 03 & 55 & 91,67 & 5 & 8,33 & 03 & 30 & 50,00 & 30 & 50,00 \\
\hline 04 & 57 & 95,00 & 3 & 5,00 & 04 & 59 & 98,34 & 1 & 1,66 \\
\hline 05 & 59 & 98,34 & 1 & 1,66 & 05 & 56 & 93,34 & 4 & 6,66 \\
\hline 06 & 46 & 76,67 & 14 & 23,33 & 06 & 53 & 88,34 & 7 & 11,66 \\
\hline 07 & 59 & 98,34 & 1 & 1,66 & 07 & 47 & 78,34 & 13 & 21,66 \\
\hline 08 & 59 & 98,34 & 1 & 1,66 & 08 & 57 & 95,00 & 3 & 5,00 \\
\hline 09 & 57 & 95,00 & 3 & 5,00 & 09 & 53 & 88,34 & 7 & 11,66 \\
\hline 10 & 42 & 70,00 & 18 & 30,00 & 10 & 54 & 90,00 & 6 & 10,00 \\
\hline 11 & 43 & 71,67 & 17 & 28,33 & 11 & 58 & 96,67 & 2 & 3,33 \\
\hline 12 & 57 & 95,00 & 3 & 5,00 & 12 & 57 & 95,00 & 3 & 5,00 \\
\hline 13 & 58 & 96,67 & 2 & 3,33 & 13 & 58 & 96,67 & 2 & 3,33 \\
\hline 14 & 56 & 93,34 & 4 & 6,66 & 14 & 59 & 98,34 & 1 & 1,66 \\
\hline 15 & 52 & 86,67 & 8 & 13,33 & 15 & 56 & 93,34 & 4 & 6,66 \\
\hline 16 & 51 & 85,00 & 9 & 15,00 & 16 & 45 & 75,00 & 15 & 25,00 \\
\hline 17 & 54 & 90,00 & 6 & 10,00 & 17 & 55 & 91,67 & 5 & 8,33 \\
\hline 18 & 60 & 100,00 & 0 & 0,00 & 18 & 55 & 91,67 & 5 & 8,33 \\
\hline 19 & 55 & 91,67 & 5 & 8,33 & 19 & 28 & 46,67 & 32 & 53,33 \\
\hline 20 & 57 & 95,00 & 3 & 5,00 & 20 & 53 & 88,34 & 7 & 11,66 \\
\hline \multirow[t]{3}{*}{21} & 56 & 93,34 & 4 & 6,66 & 21 & 53 & 88,34 & 7 & 11,66 \\
\hline & & & & & 22 & 52 & 86,67 & 8 & 13,33 \\
\hline & & & & & 23 & 56 & 93,34 & 4 & 6,66 \\
\hline
\end{tabular}

Legenda: GND = grupo não-disfônico; GD = grupo disfônico

Tabela 3. Comparação entre as médias dos valores do Teste de Detecção de Padrão de Frequência e do Teste de Detecção de Padrão de Duração, de acordo com os achados nos grupos não-disfônico e disfônico

\begin{tabular}{|c|c|c|c|c|c|}
\hline \multirow{2}{*}{ Variável } & \multicolumn{2}{|c|}{ GND } & \multicolumn{2}{|c|}{ GD } & \multirow{2}{*}{ Valor de $p$} \\
\hline & AC_N & AC_P & AC_N & AC_P & \\
\hline TDPF & $58,57 \pm 1,96$ & $97,62 \% \pm 3,27$ & $56,17 \pm 3,83$ & $93,62 \% \pm 6,39$ & $0,014^{*}$ \\
\hline TDPD & $54,43 \pm 5,08$ & $90,72 \pm 8,46$ & $50,00 \pm 10,64$ & $83,34 \pm 17,73$ & 0,084 \\
\hline
\end{tabular}

*Valores significativos $(p \leq 0,05)$ - Teste $t$ de Student

Legenda: TDPF = Teste de Detecção de Padrão de Frequência; TDPD = Teste de Detecção de Padrão de Duração; AC_N = número de acertos; $A C \_P=$ porcentagem de acertos; GND = grupo não-disfônico; GD = grupo disfônico

vocal do tempo, da qualidade e da direção. Estudos sugerem que as áreas auditivas corticais direitas podem utilizar diferentes mecanismos para realizar o fechamento do pitch em sons cuja frequência fundamental foi filtrada, desse modo, indicam que o processamento neural do pitch é diferente nos hemisférios direito e esquerdo, o que permite ao sistema audiovocal detectar variações temporais e espectrais por meio do feedback auditivo e consequentemente, do que permitirá o controle do pitch vocal $^{(27)}$.

Esta é uma das razões pela qual o professor, em geral, apresenta a intensidade de sua voz excedente aos padrões normais. $\mathrm{O}$ professor passa grande parte de seu dia em ambientes ruidosos. Estudos realizados no Brasil ${ }^{(15)}$ mostraram que as salas de aula apresentam nível de ruído acima dos valores determinados pela Associação Brasileira de Normas Técnicas (ABNT), o que dificulta ao professor monitorar sua produção vocal. Os ambientes não são tratados acusticamente. As janelas, pisos e paredes das escolas não favorecem a configuração de um ambiente mais silencioso, pois geralmente têm superfícies lisas que provocam reverberação, que aumenta o ruído interno ${ }^{(28)}$.

A intensidade elevada é considerada um dos maiores fatores de risco para problemas vocais. Segundo a literatura, a 
Tabela 4. Distribuição dos valores de significância encontrados em cada orelha, na relação entre o grupo de professores não-disfônicos e disfônicos, por ocasião da aplicação do teste SISI

\begin{tabular}{lcc}
\hline \multirow{2}{*}{ Intensidade (SISI) } & \multicolumn{2}{c}{ GND x GD (Valor de p) } \\
\cline { 2 - 3 } $500 \mathrm{~Hz}$ & OE & OD \\
5 incrementos de $5 \mathrm{~dB}$ & 0,186 & 0,177 \\
5 incrementos de $2 \mathrm{~dB}$ & $0,036^{*}$ & 0,185 \\
20 incrementos de $1 \mathrm{~dB}$ & $<0,001^{*}$ & $0,007^{*}$ \\
$1000 \mathrm{~Hz}$ & & \\
5 incrementos de $5 \mathrm{~dB}$ & $0,013^{*}$ & $0,002^{*}$ \\
5 incrementos de $2 \mathrm{~dB}$ & 0,088 & 0,415 \\
20 incrementos de $1 \mathrm{~dB}$ & $0,001^{*}$ & $0,039^{*}$ \\
$2000 \mathrm{~Hz}$ & & \\
5 incrementos de $5 \mathrm{~dB}$ & $0,037^{*}$ & 0,168 \\
5 incrementos de $2 \mathrm{~dB}$ & 0,578 & 0,775 \\
20 incrementos de $1 \mathrm{~dB}$ & $0,010^{*}$ & $0,007^{*}$ \\
$4000 \mathrm{~Hz}$ & & \\
5 incrementos de $5 \mathrm{~dB}$ & 0,172 & 0,366 \\
5 incrementos de $2 \mathrm{~dB}$ & 0,218 & 0,319 \\
20 incrementos de $1 \mathrm{~dB}$ & $0,001^{*}$ & $0,002^{*}$ \\
\hline
\end{tabular}

*Valores significativos $(p \leq 0,05)$ - Teste t de Student

Legenda: $\mathrm{OD}$ = orelha direita; $\mathrm{OE}$ = orelha esquerda; GND = grupo não-disfônico; GD = grupo disfônico

intensidade vocal elevada gera tensão durante a fonação e os professores não deveriam transpor para fora da sala de aula a intensidade vocal elevada usada em situação profissional, ou seja, não deveriam tornar a voz profissional um modo habitual de fala ${ }^{(28,29,30)}$.

Por outro lado, observa-se que mesmo com essas condições adversas de trabalho, muitos professores, quando em situação terapêutica para tratar dos seus problemas vocais, em pouco tempo, mostram grande evolução, enquanto outros mostram grande dificuldade. Neste estudo, pode-se observar que a percepção auditiva para traços de frequência, duração e intensidade é diferente (mesmo que diferença estatística entre os grupos não tenha sido significativa), pois se mostrou mais precisa no grupo sem disfonia. O ouvido humano é muito bom para discriminar intensidade e usa qualquer código que esteja à mão para realizar essa tarefa. Expresso em dB, a menor mudança em intensidade que produz uma diferença perceptual está entre 0,5 e 1,0 dB. Isto significa que quando um professor necessita mais do que esse valor para detectar ou identificar uma mudança no seu padrão vocal, ele pode estar apresentando uma dificuldade auditiva que pode ter impacto negativo em seu desempenho vocal.

A questão da influência da qualidade de percepção acústica na capacidade de realizar um bom feedback acústico articulatório pode ser um fator a ser considerado nestes dois grupos ora analisados, e outras pesquisas, até mesmo com outras categorias de profissionais da voz, são necessárias para entender melhor esse fenômeno.
Tabela 5. Distribuição dos valores de significância encontrados nos grupos não-disfônico e disfônico, na relação entre as duas orelhas, por ocasião da aplicação do teste SISI

\begin{tabular}{llc}
\hline \multirow{2}{*}{ Intensidade (SISI) } & \multicolumn{2}{c}{ OD x OE (Valor de $\mathrm{p})$} \\
\cline { 2 - 3 } $500 \mathrm{~Hz}$ & $\mathrm{GND}$ \\
\hline 5 incrementos de $5 \mathrm{~dB}$ & 0,180 & 0,414 \\
5 incrementos de $2 \mathrm{~dB}$ & 0,722 & 0,457 \\
20 incrementos de $1 \mathrm{~dB}$ & 1,000 & 0,622 \\
$1000 \mathrm{~Hz}$ & & \\
5 incrementos de $5 \mathrm{~dB}$ & 0,914 & 0,317 \\
5 incrementos de $2 \mathrm{~dB}$ & 0,692 & 0,343 \\
20 incrementos de $1 \mathrm{~dB}$ & 0,461 & 0,676 \\
$2000 \mathrm{~Hz}$ & & \\
5 incrementos de $5 \mathrm{~dB}$ & 0,493 & 0,357 \\
5 incrementos de $2 \mathrm{~dB}$ & 0,243 & $0,023^{*}$ \\
20 incrementos de $1 \mathrm{~dB}$ & 0,915 & 0,322 \\
$4000 \mathrm{~Hz}$ & & \\
5 incrementos de $5 \mathrm{~dB}$ & 0,096 & 0,102 \\
5 incrementos de $2 \mathrm{~dB}$ & 0,855 & 0,905 \\
20 incrementos de $1 \mathrm{~dB}$ & 0,655 & $0,036^{*}$ \\
\hline
\end{tabular}

*Valores significativos $(p \leq 0,05)$ - Teste t de Student

Legenda: $\mathrm{OD}$ = orelha direita; $\mathrm{OE}$ = orelha esquerda; $\mathrm{GND}$ = grupo não-disfônico; $\mathrm{GD}=$ grupo disfônico

\section{CONCLUSÃO}

A análise relacionada à percepção auditiva mostra diferença apenas para o parâmetro da frequência entre professores disfônicos e não disfônicos. Estudos com maior número de sujeitos e com outros testes que avaliem o limiar diferencial de intensidade podem melhor esclarecer a relação entre problemas de voz e dificuldades na percepção de pequenas diferenças de intensidade.

\section{REFERÊNCIAS}

1. Roy N, Merril RM, Thibeault S, Gray SD, Smith EM. Voice disordes in teachers and the general population; effects on work performance, attendance, and future career choices. J Speech Lang Hear Res. 2004Jun;44:542-51.

2. Behlau M, Zambon F, Guerrieri AC, Roy N. Panorama Epidemiológico sobre a voz do professor no Brasil. In: $17^{\circ}$. Congresso Brasileiro de Fonoaudiologia. I Congresso IberoAmericano de Fonoaudiologia. Anais Rev Soc Bras Fonoaudiol. [Internet]. Suplemento 2009: 1511. [citado 2011 Nov 15]. Disponível em: http://www.sbfa.org.br/portal/anais2009/anais_select. php?op=PR\&cid=1511\&tid=1

3. Dragone MLOS. Programa de saúde vocal para educadores: ações e resultados. Rev CEFAC. 2011;13(06):1133-43.

4. Ishii C, Arashiro PM, Pereira LD. Ordenação e resolução temporal em cantores profissionais e amadores afinados e desafinados. Pro Fono. 2006;18(3):285-92. 
5. Ferreira LP, Giannini SPP, Figueira S, Silva EE, Karmann DF, Thomé de Souza TM. Condições de produção vocal de professores da Prefeitura do Município de São Paulo. Distúrb Comun. 2003;14(2):275-307.

6. Servilha EAM, Pena J. Tipificação de sintomas relacionados à voz e sua produção em professores identificados com ausência de alteração vocal na avaliação fonoaudiológica. Rev CEFAC. 2010MaioJun;12(3)454-61.

7. Penteado RZ. Relações entre saúde e trabalho docente: percepções de professores sobre saúde vocal. Rev Soc Bras Fonoaudiol. 2007JanMar;12(1):18-22.

8. Fortes FSG, Imamura, RT, Domingos HT, SennesUL. Perfil dos profissionais da voz com queixas vocais atendidos em um centro terciário de saúde. Rev Bras Otorrinolaringol. 2007Fev;73(1):27-31.

9. Silvério CAK, Gonçalves OGC, Penteado ZR, Vieira TPG, Libardi A, Rossi D. Ações em saúde vocal: proposta de melhoria do perfil vocal de professores. Pro Fono. 2008Jul-Set;20(3):177-82.

10. Choi-Cardim K, Behlau M, Zambon F. Sintomas vocais e perfil de professores em um programa de saúde vocal. Rev CEFAC. 2010SetOut;12(5):811-19.

11. Jardim R, Barreto MS, Assunção AA. Condições de Trabalho, qualidade de vida e disfonia entre docentes. Cad Saúde Pública. 2007Out;23(10): 2439-61.

12. Grillo MHMM, Penteado RZ. Impacto da voz na qualidade de vida de professore(a)s do ensino fundamental. Pro Fono. 2005SetDez;17(3) 311-20.

13. Simberg S, Sala E, Vehmas K, Laine A. Changes in the prevalence of vocal symptoms among teachers during a twelve-year period. J Voice. 2005Mar;19(1):95-102.

14. Gonçalves VSB, Silva LB, Coutinho AS. Ruído como agente comprometedor da inteligibilidade de fala dos professores. Prod. 2009;19(3): 466-76

15. Medeiros AM, Barreto SM, Assunção AA. Voice disorders (dysphonia) in public school female teachers working in Belo Horizonte: prevalence and associated factors. J Voice. 2008Nov;22(6):676-87.

16. Eniz A O. Poluição sonora em escolas do Distrito Federal [dissertação]. Brasília: Universidade Católica de Brasília, Programa de Pós-Graduação Stricto Sensu em Planejamento e Gestão Ambiental; 2004.

17. Servilha EAM, Ruela IS. Riscos ocupacionais à saúde e voz de professores: especificidades das unidades de rede municipal de ensino. Ver CEFAC. 2010Fev;12(1):109-14.
18. Cavadas M, Pereira LD, Behlau M. Disfonia Infantil e Processamento Auditivo Central. In: Valle MGM. Voz: diversos enfoques em Fonoaudiologia. Rio de Janeiro: Revinter; 2002. p.99-109.

19. Auditec OS. Manual de aplicação do Pitch Pattern Sequence Test e Duration Pattern Sequence Test. Saint Louis, 1997.

20. Momensohn-Santos T, Russo ICP. A prática da audiologia clínica. $4^{\mathrm{a}}$. ed. São Paulo: Cortez, 2005.

21. Pereira Filho R. Avaliação dos processos auditivos temporais em adultos disfônicos funcionais [monografia especialização]. São Paulo: Universidade Federal de São Paulo; 1999.

22. Dejonchere PH, Bradley P, Clemente P, Cornut G, CrevierBuchman L, Friedrich G, et al. A basic protocol for funcional assement of voice pathology, specially for investigating the efficacy of (phonosurgical) treatments and evaluating new assement techniques. Guideline elaborated by the Committee on Phoniatrics of the European Laryngological Society (ELS). Eur Arch Othoryinolaringol. 2001;258:77-82.

23. Corazza MCA. Avaliação do processamento auditivo central em adultos: teste de padrões tonais auditivos de frequência e testes de padrões tonais auditivos de duração [tese]. São Paulo: Universidade Federal de São Paulo; 1998.

24. Luchesi KF, Moura LF, Kitamura S, Nakamura HY. Problemas vocais no trabalho: prevenção na prática docente sob a óptica do professor. Saúde Soc. 2009;18(4):673-81.

25. Dreossi RCF, Momensohn-Santos T. O ruído e sua interferência sobre estudantes em uma sala de aula: revisão de literatura. Pro Fono. 2005; 17(2):251-8.

26. Libardi A, Gonçalves CGO, Vieira TPG, Silvério KCA, Rossi D, Penteado RZ. O ruído em sala de aula e a percepção dos professores de uma escola de ensino fundamental de Piracicaba. Distúrb Comun. 2006;18(2):167-78.

27. Behroozmand R, Korzyukov O, Larson CR. ERP Correlates of pitch error detection in complex tone and voice auditory feedback with missing fundamental. Brain Res. 2012Apr;1448:89-100.

28. Perkell JS, Guenther FH, Lane H, Mattes ML, Perrier P, Vick J, et al. A theory of speech motor control and supporting data from speakers with normal hearing and with profound hearing loss. J Phonetics. 2000Jul;28(3):233-72

29. Rodrigues N. Neurolinguística dos distúrbios da fala. $3^{\text {a }}$. ed. São Paulo: Cortez, 1999. 200p.

30. Simões M, Latorre MRDO. Prevalência de alteração vocal em educadoras e sua relação com a auto-percepção Rev Saúde Pública. 2006;40(6):1013-8. 